Indian J. Anim. Hlth. (2020), 59(1) : 97-98

DOI:10.36062/ijah.59.1.2020.97-98

Short Communication

\title{
ISOLATION OF FLUCONAZOLE RESISTANT ALTERNARIA ALTERNATA FROM AN INDIAN PARROT
}

\author{
A. D. SINGH ${ }^{* 1}$, C. DEBNATH ${ }^{1}$, K. BATABYAL ${ }^{2}$ AND A. BANERJEE ${ }^{2}$ \\ ${ }^{1}$ Department of Veterinary Public Health, West Bengal University of Animal and Fishery Sciences, \\ 37, K. B. Sarani, Kolkata-700 037, West Bengal, India \\ ${ }^{2}$ Department of Veterinary Microbiology, West Bengal University of Animal and Fishery Sciences, \\ Kolkata-700 037, West Bengal, India
}

A 2-year-old parrot was presented with a feather picking habit for the last 8 days. Skin scraping revealed fungal infection and further confirmation was done through sequencing the ITS gene of rRNA region. Antifungal susceptibility testing with six commonly used antifungals revealed resistance against fluconazole. To the best of author's knowledge, this is the first report of fluconazole resistant Alternaria alternata infection from Indian parrot.

Key words: Alternaria alternata, Antifungal resistance, ITS, Terbinafine

Alternaria alternata is a contagious pathogenic fungus which has been most studied fungus until now because of its association with infection in plants, animals and humans (Singh and Denning, 2012; Raza et al., 2015). The fungus has been known to cause hypersensitivity, pneumonitis, allergic sinusitis, bronchial asthma and sometimes disseminated infection in humans (Singh and Denning, 2012; Hattab et al., 2019). In dogs, Alternaria alternata was found to be associated with onychorrhexis and black plaque in oral mucosa (Samanta, 2015).

Until now there are no published reports of Alternaria alternata infection available in Indian parrot. In this study, a 2-year old parrot was presented to the hospital with a feather picking habit for last 8 days. This had resulted in itching, redness and loss of feathers from the site of the lesion (Fig. 1). According to the owner this habit developed suddenly. Parrot was given a liquid supplementation of multivitamins (vitamin A, vitamin D3, vitamin E and Bcomplex), minerals (calcium, selenium, zinc, magnesium and sulphur) and fatty acids (Omega-3 and Omega-6) to avoid nutritional deficiencies. The parrot also had a regular exposure to fresh air and direct sunlight for 30 minutes.

The tentative diagnosis was done as bacterial infection, so mupirocin ointment $(2 \% \mathrm{w} / \mathrm{w})$ $\left(\right.$ Mupinase $^{\mathrm{TM}}, \mathrm{Cipla}^{\circledR}$ ) was advised twice daily for 5 days. As no improvement was noticed, samples were taken for detection of parasitic and fungal infection if any. Parasitic infection was negative for mange while fungal culture incubated on Sabouraud's Dextrose Agar at $25^{\circ} \mathrm{C}$ for 2 weeks showed a growth. The colony was dark gray and fluffy with white concentric circle (Fig. 3a) and microscopic examination with Lactophenol cotton blue stain, revealed septate hyphae along with dictyoconidia in chains (Fig. 3b). With these conventional 
Indian Journal of Animal Health, June, 2020

\section{Alternaria alternata in a parrot}

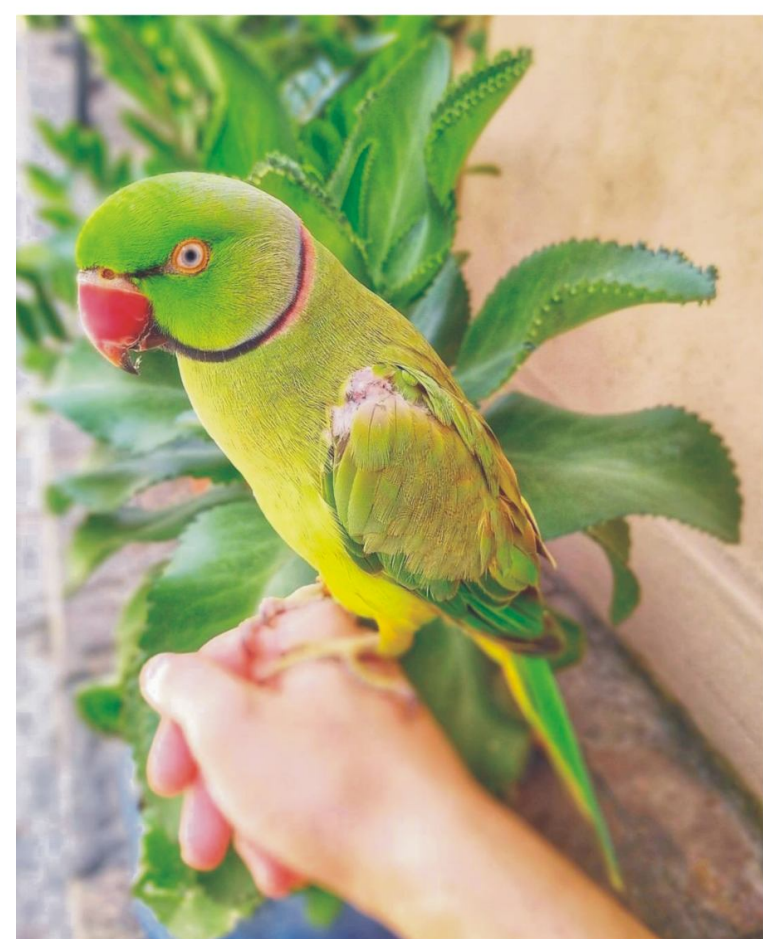

Fig. 1. Skin lesion in a parrot showing loss of feathers and redness

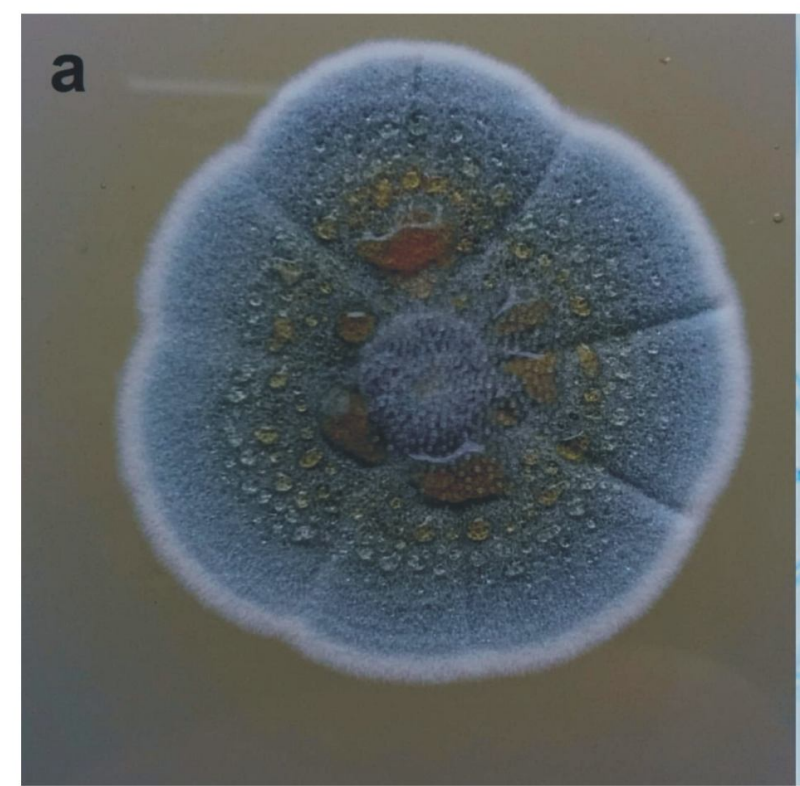

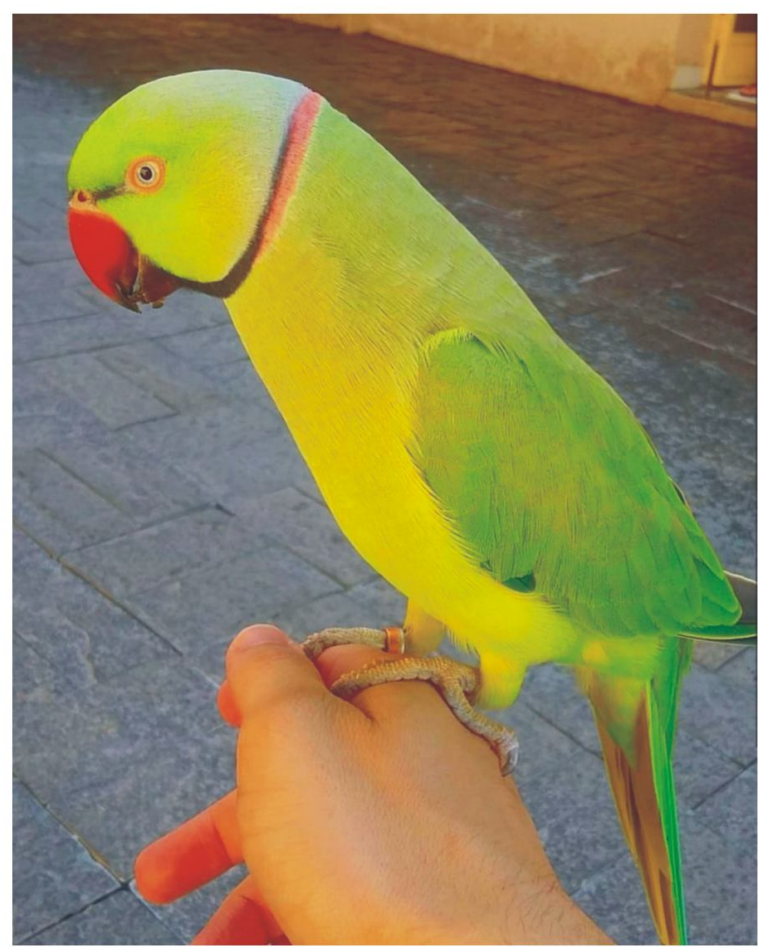

Fig. 2. Growth of feathers after successful treatment with topical terbinafine (1\% cream)

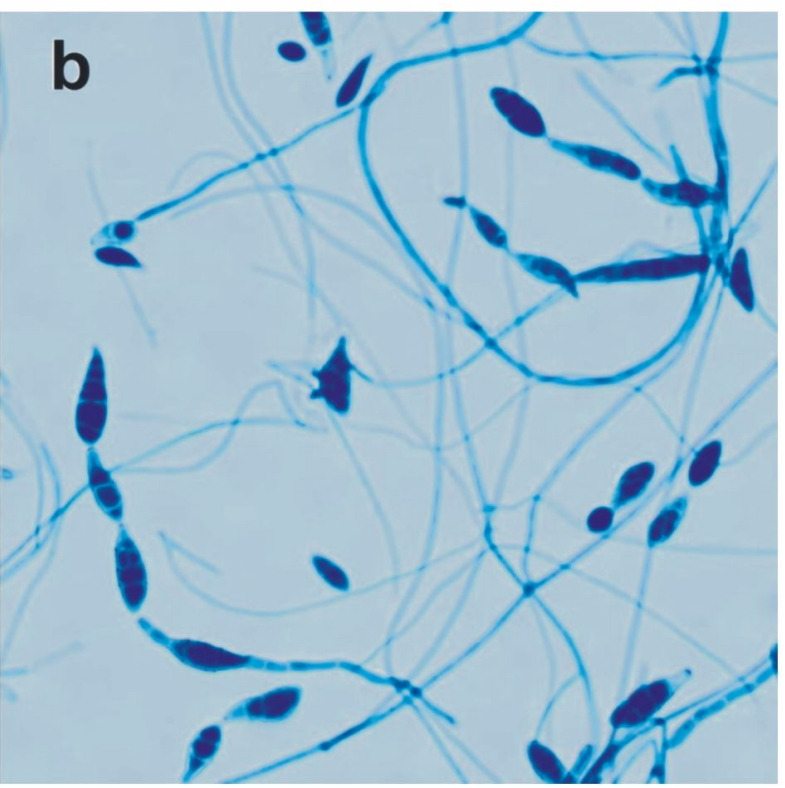

Fig. 3. Obverse section of Alternaria alternata growth on SDA medium (a) and lactophenol cotton blue staining showing septate hyphae and dictyoconidia in chains under phase contrast microscope (b) (100X) 
morphological characteristics, the fungus was identified as Alternaria alternata. Further confirmation was determined by sequencing the ITS gene of rRNA region. The gene sequence has been submitted to GenBank database under accession number MT313680. Antifungal susceptibility testing was performed by using Broth Micro-dilution Assay method according to standard protocol given by Clinical Laboratory Standard Institute against six commonly used antifungals with few modifications (Singh et al., 2018). The test was done in triplicates.

Minimum inhibitory concentration (MIC) value of fluconazole was $32 \mu \mathrm{g} / \mathrm{mL}$, while MIC's for itraconazole, clotrimazole, miconazole, terbinafine, and amphotericin-B were 4, 2, 2, 0.5 and $8 \mu \mathrm{g} / \mathrm{mL}$, respectively. High resistance was exhibited against fluconazole. Topical terbinafine ( $1 \%$ cream) (Terbicip ${ }^{\mathrm{TM}}$, Cipla ${ }^{\circledR}$ ) was selected for the treatment as the isolate was sensitive and the drug was easily available in the locality. The owner was advised to apply the cream twice daily for 14 days. To see the effect of selected antifungal agent on the lesion, samples were again taken on $7^{\text {th }}$ and $14^{\text {th }}$ days post-treatment. As there was no fungal growth found on SDA media on both the dates, mycological cure was obtained alongside the

\section{REFERENCES}

Hattab Z, Lasfar NB, Abid M, Bellazreg F, Fathallah A et al., 2019. Alternaria alternata infection causing rhinosinusitis and orbital involvement in an immunocompetent patient. New Microbes New Infect, 32: 1-2

Raza H, Khan RU, Anwar K and Muhammad K, 2015. Visceral phaeohyphomycosis caused by Alternaria alternata offering a diagnostic as well as a therapeutic challenge. Saudi J Kidney Dis Transpl, 26(2): 339-343

Samanta, I, 2015. Veterinary Mycology, Berlin, Germany, Springer, pp135 site of the lesion became normal (Fig. 2). To remove the remains of infectious agent the cream was continued till $14^{\text {th }}$ day. No side effects were observed in the parrot during treatment.

To the author's best knowledge, this is the first report on isolation of fluconazole resistant Alternaria alternata from an Indian parrot. This fungus causes diseases in various fruits, vegetables and plants, which might be a source of infection in birds, especially caged birds since they have an unlimited supply of these foods in their cage (Troncoso-Rojas and Tiznado-Hernandez, 2014). This in fact can increase the chance of infection in immunocompromised individuals having these pets. Further studies should be undertaken in this context to evaluate the potentiality of this virulent pathogen.

Conflict of interest: The authors declare no conflict of interest.

\section{ACKNOWLEDGEMENT}

The authors express their sincere gratitude to Indian Council of Agricultural Research (ICAR), New Delhi, India for providing the necessary funds under "Outreach Programme on Zoonotic Diseases".

Singh A, Debnath C, Banerjee A, Batabyal K, Roy B et $a l ., 2018$. Effects of propylene glycol and magnesium chloride against dermatophytes isolated from companion animals. Indian J Anim Hlth, 57(2): 213-218

Singh B and Denning DW, 2012. Allergic bronchopulmonary mycosis due to Alternaria: Case report and review. Med Mycol Case Rep, 1(1): 20-23

Troncoso-Rojas R and Tiznado-Hernandez ME, 2014. Alternaria alternata (black rot, black spot). In Postharvest decay, Academic Press, pp147-187

Received-12.04.2020, Accepted-17.05.2020, Published-01.06.2020 